\title{
DMRT1: An Ancient Sexual Regulator Required for Human Gonadogenesis
}

\author{
David Zarkower Mark W. Murphy \\ Department of Genetics, Cell Biology, and Development, University of Minnesota, Minneapolis, MN, USA
}

\section{Keywords}

DM domain · DMRT1 · DSD · Testis

\begin{abstract}
Transcriptional regulators related to the invertebrate sexual regulators doublesex and mab-3 occur throughout metazoans and control sex in most animal groups. Seven of these $D M R T$ genes are found in mammals, and mouse genetics has shown that one, Dmrt 1, plays a crucial role in testis differentiation, both in germ cells and somatic cells. Deletions and, more recently, point mutations affecting human DMRT1 have demonstrated that its heterozygosity is associated with $46, X Y$ complete gonadal dysgenesis. Most of our detailed knowledge of DMRT1 function in the testis, the focus of this review, derives from mouse studies, which have revealed that DMRT1 is essential for male somatic and germ cell differentiation and maintenance of male somatic cell fate after differentiation. Moreover, ectopic DMRT1 can reprogram differentiated female granulosa cells into male Sertoli-like cells. The ability of DMRT1 to control sexual cell fate likely derives from at least 3 properties. First, DMRT1 functionally collaborates with another key male sex regulator, SOX9, and possibly other proteins to maintain and reprogram sexual cell fate. Second, and related, DMRT1 appears to function as a pioneer transcription factor, binding "closed" inaccessible
\end{abstract}

karger@karger.com www.karger.com/sxd

(C) 2021 S. Karger AG, Basel

Karger! chromatin and promoting its opening to allow binding by other regulators including SOX9. Third, DMRT1 binds DNA by a highly unusual form of interaction and can bind with different stoichiometries.

(c) 2021 S. Karger AG, Basel

\section{Prologue: A Brief Overview of DMRT Genes}

The $D M R T$ gene family was initially discovered in the late 1990s based on sequence similarity between products of the fruitfly sex-determining gene $d s x$ and the nematode male regulator mab-3 [Baker and Ridge, 1980; Shen and Hodgkin, 1988; Raymond et al., 1998]. These invertebrate sexual regulators encode transcription factors sharing a motif consisting of an intertwined zinc-binding module followed by an alpha-helical recognition domain that confers sequence-specific DNA binding [Erdman and Burtis, 1993; Erdman et al., 1996; Yi and Zarkower, 1999; Zhu et al., 2000; Murphy et al., 2007, 2015] (Fig. 1a, b). This bipartite motif was named the DM domain after $d s x$ and $m a b-3$, and the gene family containing the motif were christened DMRT genes, for "Doublesex- and mab-3-related transcription factors". Since $d s x$ and $m a b-3$ perform some analogous functions in sexual development and $d s x$ can partially substitute for $m a b-3$ in vivo, it appeared like- 
ly that the sequence similarity indeed represented descent from a common ancestral sexual regulator [Raymond et al., 1998]. Subsequent molecular cloning and nucleotide sequence searches have revealed that nearly all metazoan animals have DMRT genes, including some from basal clades [Chong et al., 2013; Wexler et al., 2014]. However, $D M R T$ genes are not found outside metazoans, suggesting that they arose close to the origin of multicellularity and thus potentially played an ancient role in specifying differential cell fates, sexual or otherwise.

Most animals have multiple DMRT genes, ranging from 4 in insects and 7 in mammals [Raymond et al., 1998, 1999b; Ottolenghi et al., 2002; Kim et al., 2003; Volff et al., 2003] to 11 in ctenophores such as the starlet sea anemone Nematostella, which diverged early in metazoan evolution [Wexler et al., 2014]. In addition, a number of genes with some similarity to $D M R T$ genes but lacking a functional DNA-binding domain have been identified, particularly in mammals. Some of these DMRT-like genes show sex-specific expression [Veith et al., 2006], but no functional analysis of these genes has been reported. $D M R T$ genes can play varied roles in different tissues, but in species where $D M R T$ gene function has been investigated most have at least one DMRT gene involved in sexual development, acting at the level of sex determination, sex differentiation, or, in some cases, both [Matson and Zarkower, 2012]. The functions of DMRT genes can be limited to one tissue or cell type or can be more general. For example, in mice Dmrt6 and Dmrt7 (also called $D m r t b 1$ and Dmrtc2, respectively) regulate germ cell differentiation but appear not to be required in any other cell types [Kim et al., 2007b; Zhang et al., 2014]. At the other end of the spectrum, $d s x$ controls most sexually dimorphic aspects of development in insects, regulating a wide variety of distinct functions in different cell types [Baker and Ridge, 1980; Robinett et al., 2010]. Moreover, $d s x$ is alternatively spliced to generate sex-specific Dsx protein isoforms that direct male- or female-specific differentiation throughout the body [Burtis and Baker, 1989; Robinett et al., 2010]. This distributed and diverse regulatory repertoire allows $d s x$ to act as a primary sex-determining gene in a system that, unlike sex development in vertebrates, does not involve gonadal sex hormones. Because $D M R T$ genes regulate sexual differentiation in species as divergent as nematodes [Shen and Hodgkin, 1988; Mason et al., 2008], flatworms [Chong et al., 2013], insects [Geuverink and Beukeboom, 2014], crustaceans [Kato et al., 2011], and vertebrates [Raymond et al., 2000; Matsuda et al., 2002; Smith et al., 2009; Ge et al., 2017; Webster et al., 2017], it seems likely that that sexual regulation is an an-

DMRT1 and Testis Development cestral function of this gene family [Matson and Zarkower, 2012]. As such, DMRT genes are by far the most deeply conserved regulators of sexual development found to date, and it seems likely that they have characteristic functional properties that have helped them maintain a crucial role in this process for hundreds of millions of years in organisms with diverse strategies and mechanisms of sexual development.

\section{DMRT Genes in Mammals and DMRT1 as a Conserved Sexual Regulator}

Mammals have 7 DMRT genes [Volff et al., 2003], as well as up to $3 \mathrm{X}$-linked $D M R T$-like genes encoding proteins that appear to lack a functional DM domain [Veith et al., 2006]. Mouse gene targeting has probed the functions of all 7 bona fide autosomal Dmrt genes. Mutations in 4 genes, Dmrt1, Dmrt4 (Dmrta1), Dmrt6 (Dmrtb1), and Dmrt7 (Dmrtc2), disrupt gonadal development in one or both sexes [Raymond et al., 2000; Balciuniene et al., 2006; Kawamata and Nishimori, 2006; Kim et al., 2007b; Zhang et al., 2014], confirming roles in sexual differentiation, while loss of Dmrt2 disrupts embryonic patterning [Seo et al., 2006]. Mutations in Dmrt3 and Dmrt5 (Dmrta2) mainly affect the nervous system, disrupting patterning and development of the hippocampus and cerebral cortex [Konno et al., 2012; Saulnier et al., 2013; De Clercq et al., 2018]. Dmrt3 also is important for spinal interneuron function in locomotion in mice, and variants of DMRT3 determine gait patterns in horses [Andersson et al., 2012; Vallstedt and Kullander, 2013].

Dmrt1 is the only DMRT gene shown to play a conserved role in mammalian sexual differentiation. Dmrt 1 is expressed specifically in the gonads of mice and humans, in both somatic and germline cells. In mice, Dmrt 1 mRNA is first detected in the genital ridges around E10.5 in both somatic cells and primordial germ cells (PGCs) of both sexes, and it becomes highly male-enriched by about E14.5 as testis differentiation initiates [Raymond et al., 1999a]. Similarly, DMRT1 protein is expressed from E10.5 to E11.5 in somatic and germ cells of both sexes. It becomes male-specific in somatic cells from E12.5 to E13.5, and expression continues in Sertoli cells thereafter [Lei et al., 2007]. DMRT1 is expressed in germ cells of both sexes through E13.5, disappearing by about E15.5 [Lei et al., 2007]. In males, DMRT1 is re-expressed in spermatogonia just before birth and maintained in spermatogonia subsequently [Lei et al., 2007; Matson et al., 2010]. During steady state adult spermatogenesis, DMRT1 
is expressed in mitotic cells, from Id4-gfp-expressing spermatogonial stem cells (SSCs) through differentiated B spermatogonia, disappearing in preleptotene spermatocytes that are entering meiosis [Matson et al., 2010].

Human DMRT1 expression is less fully characterized but appears similar to that of mice. DMRT1 mRNA was detected only in the testis, among 50 tissues surveyed by RNA blotting [Raymond et al., 1998]. During fetal development, DMRT1 mRNA is detectable by gestational week 11 (GW11) in both sexes. DMRT1 protein is most abundant in pre-Sertoli cells between GW10 and GW20, before becoming strong in spermatogonia from about GW24 onward [Jorgensen et al., 2012]. DMRT1 also is expressed in oogonia and oocytes prior to GW20 and downregulated upon meiotic entry, as in mice [Jorgensen et al., 2012]. In prepubertal testes, DMRT1 protein is highly expressed in spermatogonia and in pre-Sertoli cells, followed by lower expression in Sertoli cells [Jorgensen et al., 2012]. In adult testes, DMRT1 is expressed both in Sertoli cells and in spermatogonia, except in OCT2-positive A-dark cells, which are thought to represent reserve SSCs [Jorgensen et al., 2012]. Similarly, single cell RNA sequencing (scRNA-seq) has found that Dmrt1 mRNA is expressed postnatally in Sertoli cells and in SSCs, differentiating spermatogonia, and preleptotene spermatocytes, but not in meiotic and post-meiotic germ cells [Guo et al., 2018; Wang et al., 2018].

\section{Ancient Involvement of DMRT1 in Vertebrate Sex Determination and Its Evolution}

Not only is DMRT1 conserved in mammalian sex regulation but it also is a universal or near-universal sex regulator in other vertebrates. Studies in non-mammalian vertebrates have uncovered $D M R T 1$ or a close paralog controlling gonadal sex determination in species with diverse sex determination mechanisms, ranging from fish to birds to reptiles and amphibians [Matson and Zarkower, 2012; Ge et al., 2017]. Moreover, in several vertebrate clades, changes in DMRT1 appear to be associated with the acquisition of new sex determination mechanisms. In the fish Medaka, for example, sex is dominantly determined by a DMRT1 paralog called Dmy or Dmrt1bY that is located in the non-recombining region of the Y chromosome and acts analogously to $S R Y$ in mammals [Matsuda et al., 2002; Nanda et al., 2002]. Dmy and the X/Y sex chromosome pair containing it are found only in a handful of Medaka species, suggesting a comparatively recent origin for this gene variant and these sex chromosomes
[Matsuda et al., 2003; Kondo et al., 2004]. A plausible model is that Dmy formed by a duplication of Dmrt 1 that caused constitutive expression of the new gene, rendering individuals carrying it male, regardless of their genotype otherwise, and thus created a neo-Y chromosome. In birds, sex is determined by a ZZ/ZW chromosome system, with ZZ eggs developing as males and ZW eggs as females. Avian DMRT1 is located on the $\mathrm{Z}$ and missing from the W chromosome [Nanda et al., 1999]. Knockdown and overexpression experiments in chickens support the model that DMRT1 is required in 2 copies for male gonadal fate [Smith et al., 2009; Lambeth et al., 2014]. Here, a likely model is that deletion of $D M R T 1$ in an avian progenitor was associated with formation of a new sex chromosome pair. In the African clawed frog Xenopus laevis, a DMRT1 paralog, $D M-W$, is found on the $\mathrm{W}$ chromosome of a different ZW sex chromosome system [Yoshimoto et al., 2008]. In ZW individuals, DM-W dominantly interferes with function of the autosomal DMRT1 gene, preventing testis differentiation [Yoshimoto et al., 2008]. $D M-W$ has an $\mathrm{N}$-terminal DM domain but lacks some of the C-terminal sequences found in $D M R T 1$, suggesting that it formed via a partial duplication of DMRT1, resulting in a neo-W chromosome [Bewick et al., 2011]. In all of these cases it is impossible to distinguish whether a mutation affecting DMRT1 triggered the formation of new genetic sex-determining mechanisms or instead co-occurred with those events, but it does appear that DMRT1 and its close paralogs determine sex in a wide range of vertebrates and have done so for hundreds of millions of years. Function of DMRT1 in vertebrate sex determination is not limited to genetic mechanisms: in the red-eared slider turtle, DMRT1 is required for temperature-dependent sex determination, possibly via temperature-dependent methylation of the DMRT1 promoter [Ge et al., 2017]. The involvement of DMRT1 in such a breadth of sex determination systems suggests that it plays a central role in the process and has properties that make it hard to displace.

\section{DMRT1 and Disorders of Sexual Devolopment: Distal 9p Deletions Define a Critical Region}

The discovery of human DMRT1 and its mapping to 9p24.3 [Raymond et al., 1998] was of immediate relevance for disorders of sexual development (DSD) genetics. The association between monosomy of the distal short arm of chromosome $9(9 \mathrm{p})$, where DMRT1 is located, and partial or complete $46, \mathrm{XY}$ gonadal dysgenesis 
Table 1. DMRT1 microdeletions and missense mutations associated with human DSD

\begin{tabular}{|c|c|c|c|}
\hline Lesion & Phenotype & Notes & Citation \\
\hline 103-kb deletion removing DMRT1 exons 1 and 2 & 46,XY complete DSD & Hemizygous & Ledig et al. [2010] \\
\hline 35-kb deletion removing $D M R T 1$ exons 3 and 4 & 46,XY ovotesticular DSD & Hemizygous & Ledig et al. [2012] \\
\hline $\begin{array}{l}700-\mathrm{kb} \text { deletion extending distally from } 30 \mathrm{~kb} 5^{\prime} \text { of } D M R T 1 \\
\text { TSS }\end{array}$ & $\begin{array}{l}\text { 46,XY complete DSD } \\
\text { 46,XY partial DSD }\end{array}$ & Hemizygous; probands are siblings & Calvari et al. [2000] \\
\hline DMRT1 R111G missense mutation in DM domain & 46,XY complete DSD & $\begin{array}{l}\text { Heterozygous; reduced DNA } \\
\text { affinity, altered stoichiometry }\end{array}$ & Murphy et al. [2015] \\
\hline$D M R T 1$ Y84C missense mutation in DM domain & 46,XY complete DSD & Heterozygous; maternally inherited & Fan et al. [2017] \\
\hline DMRT1 R80S missense mutation in DM domain & 46,XY complete DSD & $\begin{array}{l}\text { Heterozygous; reduced DNA } \\
\text { affinity }\end{array}$ & $\begin{array}{l}\text { Buonocore et al. [2019]; } \\
\text { this paper }\end{array}$ \\
\hline DMRT1 L139Q missense mutation adjacent to DM domain & 46,XY complete DSD & Heterozygous & Chauhan et al., [2017] \\
\hline DMRT1 P74L missense mutation in DM domain & $\begin{array}{l}46, \mathrm{XY} \text { Sertoli-only } \\
\text { syndrome }\end{array}$ & $\begin{array}{l}\text { Heterozygous; reduced DNA } \\
\text { affinity }\end{array}$ & $\begin{array}{l}\text { T. Maric and M. Murphy, } \\
\text { in preparation }\end{array}$ \\
\hline
\end{tabular}

has been clear for several decades [Jotterand and Juillard, 1976; Fryns et al., 1986; Crocker et al., 1988; Hoo et al., 1988; Huret et al., 1988; Bennett et al., 1993; Ogata et al., 1997; Veitia et al., 1997, 1998]. Distal 9p monosomy also can be associated with cognitive deficits, developmental delay, and a characteristic array of dysmorphic craniofacial features [Alfi et al., 1976]. An early question was whether these effects are genetically linked, much as heterozygosity of SOX 9 causes both campomelic dysplasia and DSD [Foster et al., 1994; Wagner et al., 1994]. Identification of shorter 9p deletions narrowed the critical region for DSD to an interval on 9p24.3, near the telomere, again containing DMRT1 [McDonald et al., 1997; Flejter et al., 1998; Guioli et al., 1998; Veitia et al., 1998; Calvari et al., 2000; Ottolenghi and McElreavey, 2000]. Because these shorter deletions are found in patients with 46,XY gonadal dysgenesis but lacking the non-urogenital features of monosomy $9 \mathrm{p}$, it is clear that the gonadal and extragonadal aspects of $9 p$ deletion syndrome are genetically separable.

9p deletions consistently affect DMRT1 but most also remove other genes, including the adjacent paralog $D M R T 3$, which is immediately proximal to DMRT1 on $9 \mathrm{p}$ [Ottolenghi et al., 2000]. The co-involvement of DMRT3 in so many DSD-associated $9 \mathrm{p}$ deletions raised the possibility that gonadal dysgenesis might result from combined hemizygosity of DMRT1 with this gene. This possibility cannot be definitively excluded but appears unlikely on several grounds. First, although both genes are expressed in the fetal gonad, targeted deletion of Dmrt3 (and the nearby Dmrt2) in the mouse has not revealed a function in gonadal development [Seo et al., 2006; De Clercq et al., 2018; Desmaris et al., 2018]. Moreover, deletion of the interval containing Dmrt1 and Dmrt3 in mice, either using CRISPR/Cas9 [Inui et al., 2017] or by meiosis-mediated recombination between loxP sites (D.Z., unpubl. data), does not cause a more severe phenotype than mutation of Dmrt1 alone. The most compelling line of evidence is the identification of DSD cases involving 9p microdeletions and point mutations that affect only DMRT1. At least 3 hemizygous microdeletions have been reported that likely affect just $D M R T 1$ (Table 1 ). One, a deletion of $103 \mathrm{~kb}$ in a patient with $46, \mathrm{XY}$ complete gonadal dysgenesis, removed exons 1 and 2 of DMRT1 [Ledig et al., 2010], while a second deletion of $35 \mathrm{~kb}$, associated with 46,XY ovotesticular DSD, removed exons 3 and 4 of DMRT1 [Ledig et al., 2012]. A 3rd deletion was found in two 46,XY sisters, one with complete gonadal dysgenesis and female external genitalia and the other with partially virilized DSD (ovotestes and virilized female external genitalia) [Calvari et al., 2000]. This deletion removed an interval of $700 \mathrm{~kb}$ located distal to $D M R T 1$ but extending within about $30 \mathrm{~kb}$ of the DMRT1 transcriptional start site, and thus may have disrupted $D M R T 1$ upstream regulatory elements. The difference in DSD severity between these 2 sisters helps address the long-standing question of why $9 \mathrm{p}$ deletion-associated DSD is variable. The degree of DSD can range from male genitalia with small dysgenic testes to complete gonadal DSD with sex reversal and does not correlate in an obvious way with the extent of the $9 \mathrm{p}$ deletion. In addition, there has been no indication of loss of DMRT1 heterozy- 


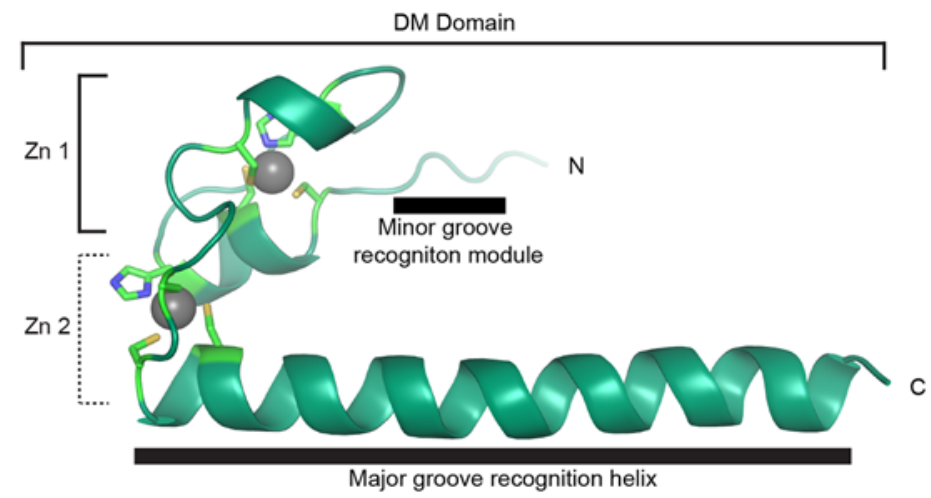

b

b Minor groove recogniton module Zn 1 Mutations: R80S Y84C $\mathrm{Zn} 2$ ........... Major groove recognition helix

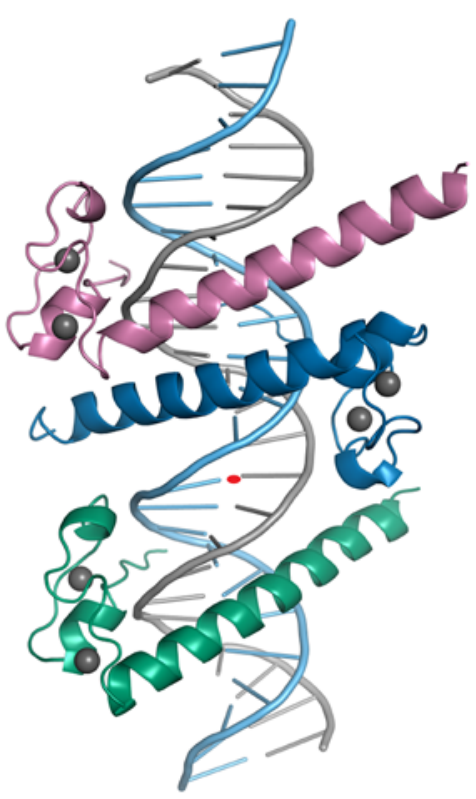

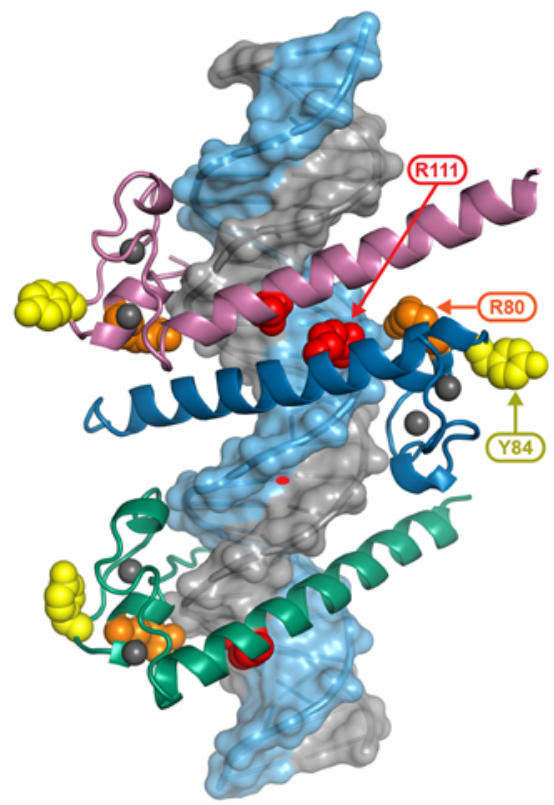

Fig. 1. The DM domain and DMRT1/DNA interaction. a X-ray crystal structure of the human DMRT1 DM domain from Murphy et al. [2015] with major structural domains indicated. b Conservation of metazoan DM domains. Positions of structural domains and sites of human mutations associated with DSD are indicated. c X-ray crystal structure of human DMRT1 DM domains bound to DNA from Murphy et al. [2015], with DMRT1 protomers in distinct colors. Two protomers (pink and blue) insert in the DNA major groove on one side of the binding site, while a single pro- tomer (green) inserts in the major groove on the other side. Red oval indicates central basepair of the punctuated pseudopalindromic DNA site. $\mathbf{d}$ DM domain amino acids affected by human DMRT1 point mutations associated with DSD. Affected amino acid side-chains are shown as space-filling models and labeled. R80 makes contact with the DNA phosphate backbone, Y84 is in the zinc module, and R111 makes contacts with conserved DNA bases in the recognition site. 
gosity in 9p-associated DSD. Given the range of severity and the finding that siblings with the same deletion can have distinct DSD phenotypes, it is more likely that variable penetrance of distal 9p deletions reflects interaction between DMRT1 hemizygosity and genetic background, rather than indicating a contiguous gene syndrome or a difference in functional DMRT1 copy number.

While distal $9 \mathrm{p}$ clearly is required in 2 copies for normal male gonadogenesis, it is less clear whether it plays a role in human ovarian development or function. On the one hand, primary hypogonadism and premature ovarian failure have been reported in 46,XX individuals with terminal 9p deletions [Muroya et al., 2000; Ogata et al., 2001; Fujimoto et al., 2004; Bartels et al., 2013], suggesting a significant role for this region in female gonadal development and function. On the other hand, the maternal inheritance of 9p deletions [Calvari et al., 2000; Quinonez et al., 2013] as well as reports of 46,XX fetuses and adults with distal 9p deletions but apparently normal ovaries [Vialard et al., 2002; Fujimoto et al., 2004; Ounap et al., 2004 ] suggest that distal 9p hemizygosity does not preclude ovarian differentiation and oogenesis. In sum, it appears that one or more dose-sensitive loci on distal 9p, potentially including DMRT1, play a significant but not essential role in ovarian differentiation.

\section{DMRT1 Point Mutations Confirm Its Role in Male Gonadogenesis}

Despite DNA sequencing efforts by a number of laboratories, DMRT1 point mutations associated with DSD or male infertility that convincingly cause loss of gene function have been surprisingly rare [Raymond et al., 1999b; Calvari et al., 2000; Ottolenghi et al., 2000; Tewes et al., 2014; Eggers et al., 2016]. Nonetheless, exome sequencing has so far detected 4 heterozygous point mutations in 46,XY individuals with complete gonadal dysgenesis that are likely to reduce DMRT1 function (Table 1). All 4 mutations affect amino acids in or adjacent to the DM domain (Fig. 1b). One de novo mutation, R111G [Murphy et al., 2015], alters a highly conserved amino acid that makes specific hydrogen-bond contacts with bases in the DNA binding consensus site and also coordinates G118 to help stabilize the DM domain tertiary structure. Substitution with alanine at this position nearly eliminates in vitro DNA binding, confirming the importance of this residue [Murphy et al., 2015]. Substitution of R111 to G severely reduces DNA binding affinity of the mutant protein but also allows it to alter the

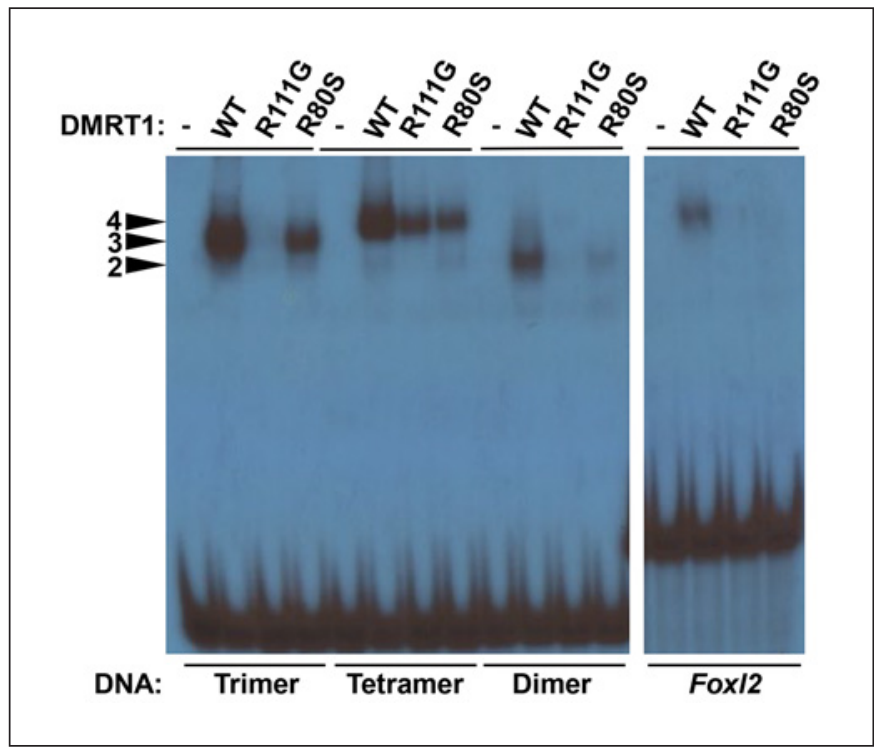

Fig. 2. Sex-reversing R111G and R80S mutations reduce DNA binding by DMRT1 in vitro. Left panel: DMRT1 protein translated in vitro was incubated with radiolabeled double-stranded DNAs previously shown to bind dimers, trimers, or tetramers of DMRT1 as previously described [Murphy et al., 2015]. Reactions contained unprogrammed reticulocyte lysate $(-)$, lysate programmed with wild-type synthetic human Dmrt1 mRNA (WT), or synthetic mRNA encoding the R111G or R80S mutant DMRT1 proteins, as indicated. After incubation, reactions were subjected to native gene electrophoresis and autoradiography. Mobility of dimers, trimers, and tetramers are indicated by arrowheads. The R111G mutation reduced trimer and dimer binding more severely than tetramer binding, while the R80S mutation reduced dimer and tetramer binding more severely than trimer binding. Right panel: DMRT1 proteins were incubated with DNA containing a DMRT1 binding site from the Foxl2 locus. Both mutations severely reduced binding to this site.

stoichiometry of binding by wild-type protein in vitro, suggesting that R111G may function as a dominant negative mutation [Murphy et al., 2015]. A second mutation, Y84C, alters a position in the zinc-binding module of the DM domain [Fan et al., 2017]. Mutations at this position have not been observed in model organisms or tested in vitro, but the introduction of a cysteine is likely to disrupt folding of the zinc-binding module and consequently affect DNA affinity. The Y84C mutation was maternally inherited, further indicating that $D M R T 1$ heterozygosity can be compatible with female fertility. The 3rd mutation, a de novo R80S change, alters a conserved residue in the DM domain that makes hydrogen-bond contacts with the DNA backbone [Murphy et al., 2015; Buonocore et al., 2019]. Molecular dynamics simulation predicted that this change should reduce DNA affinity [Buonocore 
et al., 2019]. To directly test that prediction, we have compared in vitro DNA binding affinity of full-length human wild type, R80S-, and R111G-substituted DMRT1 proteins to labeled synthetic DNA oligonucleotides by electrophoretic mobility shift analysis (EMSA) (Fig. 2). The R80S mutant protein bound less efficiently than the wild type. As discussed below, DMRT1 can bind DNA as a dimer, trimer, or tetramer. R80S mutant DMRT1 binding was reduced less severely than that of R111G DMRT1 on DNAs that bind DMRT1 trimers and dimers but more severely on a site that binds DMRT1 tetramers. Both mutant proteins showed severely reduced binding on a DMRT1 tetramer site from the Foxl2 locus. Collectively, the data were consistent with the R80S mutation being responsible for DSD in the affected patient. The 4th mutation, $\mathrm{L} 139 \mathrm{Q}$, is a de novo change affecting a conserved residue adjacent to the DM domain [Chauhan et al., 2017]. This residue is not included in the published $\mathrm{X}$-ray structure, it has not been altered in model organism genetic screens, and its role in DNA binding has not yet been tested; thus, how the L139Q mutation affects DMRT1 function remains to be established. In addition, a heterozygous P74L mutation has been found in 2 brothers with Sertoli-only syndrome. It is predicted to affect minor groove recognition and reduces DMRT1 DNA binding affinity, suggesting that reduced DMRT1 function also can cause infertility without DSD (T. Maric and M. Murphy, in preparation).

A handful of other DMRT1 mutations or rare variants outside the DM domain have been reported in DSD [Raymond et al., 1999b] and male infertility cases [Tewes et al., 2014]. It is possible that these variants represent lossof-function mutations, but given the lower sequence conservation of the affected regions and the lack of a simple and robust functional assay for DMRT1, it cannot yet be excluded that these are merely uncommon neutral variants. The low discovery rate for DMRT1 mutations in DSD and the absence of homozygous DMRT1 mutations is notable. One suggested explanation is that DMRT1 deletions also are associated with male infertility, allowing the load of DMRT1 mutations to be purged by spermatogenic failure [Lopes et al., 2013].

\section{Insights into DMRT1 from Mouse Conditional Genetics: DMRT1 and Spermatogenesis}

Conditional gene targeting technology in mice has allowed a detailed dissection of Dmrt1 function in a mammal with similar reproductive biology to that of humans.
Conditional genetics has confirmed the importance of Dmrt1 in gonadal development has found similarities and differences to its function in humans and has uncovered some unexpected roles for DMRT1. In the mouse, DMRT1 plays a major role in male but not female gonadal development, as appears to be the case in humans [Raymond et al., 2000]. However, in mice the Dmrt1 gene is fully recessive rather than haploinsufficient. This difference in dose-sensitivity is not unusual for genes involved in mouse and human sex regulation. For example, SOX9, WT1, and SF1 are haploinsufficient for human male gonadogenesis but recessive for testis differentiation in the mouse [Parker et al., 1999; Barrionuevo et al., 2006]. Homozygous deletion of Dmrt1 in XX mice causes a deficit in primordial follicles but does not significantly reduce female fertility [Krentz et al., 2011]. By contrast, XY Dmrt1 homozygous mutants have severely dysgenic gonads [Raymond et al., 2000]. However, XY homozygous Dmrt1 mutant mice still undergo male nongonadal development, in contrast to 46 ,XY humans with heterozygous $D M R T 1$ point mutations or deletions, who can have complete gonadal dysgenesis at an early enough stage to cause complete male-to-female phenotypic sex reversal. Indeed, testicular development is superficially normal in D mrt $1^{-/-}$mice of mixed genetic background until birth, with gonadal dysgenesis becoming apparent mainly between 1 and 4 weeks postnatally [Raymond et al., 2000; Fahrioglu et al., 2007; Kim et al., 2007a]. It therefore appears that there are fundamental differences, at least in timing, between the requirements for $D M R T 1$ in human and Dmrt1 in mouse. While these differences must be kept in mind, the mouse has nonetheless been useful for deeper exploration of DMRT1 function than is possible in humans.

Conditional gene targeting has allowed the dissection of requirements for Dmrt1 in germ cells and Sertoli cells and has revealed distinct functions in different cell types and at different stages of development. The role of Dmrt 1 in the germ line has been reviewed in detail elsewhere [Zarkower, 2013; Zhang and Zarkower, 2017] and will be summarized briefly here. Consistent with its expression in fetal PGCs and postnatal spermatogonia, Dmrt1 is required both for the establishment of postnatal spermatogenesis and subsequently for the maintenance of the progenitor pool that supplies adult steady state spermatogenesis [Fahrioglu et al., 2007; Kim et al., 2007a]. Fetal loss of Dmrt1 blocks perinatal mitotic reactivation, migration, and viability of prospermatogonia [Kim et al., 2007a]. Later, in steady-state adult spermatogenesis, Dmrt1 has multiple distinct roles affecting spermatogonial stem 
cells: it activs the stem cell regulator Plzf to help maintain the SSC pool and it is required also in Ngn3-positive committed progenitor cells to let them replenish the SSC pool when they are artificially depleted [Zhang et al., 2016]. In steady state spermatogenesis, Dmrtl also has a key role in differentiating spermatogonia. In these cells, rather than promoting self-renewal, Dmrt1 promotes differentiation and proliferation by activating genes including Sohlh 1 and inhibits premature meiotic initiation by inhibiting retinoic acid signaling and repressing Stra8 [Matson et al., 2010]. Normally meiotic initiation is triggered in part by degradation of DMRT1 via a $\beta$-TrCP ubiquitin ligase complex [Nakagawa et al., 2017]. The importance of this regulation is revealed when $\beta-\operatorname{TrCP}$ is depleted, which stabilizes DMRT1 in preleptotene cells, reduces STRA8 expression in these cells, and prevents meiosis. The suite of germline DMRT1 functions collectively allows spermatogonia to proliferate and differentiate, supported by a stable stem cell pool, and promotes long-term fertility. How DMRT1 activity is modified to perform different roles at different stages of spermatogonial development is important but just beginning to be understood, as discussed below.

\section{Insights from Mouse Conditional Genetics: DMRT1 and Cancer}

DMRT1 not only controls normal spermatogenesis but also is linked to germ cell cancer in both mice and humans. In mice of mixed or C57BL/6J genetic background, the Dmrt1 mutant testis appears normal until shortly after birth, but in male mice of the $129 \mathrm{~Sv} / \mathrm{J}$ inbred genetic background, mutant fetal germ cells fail to undergo cell cycle arrest and downregulation of pluripotency regulators, and this results in a very high incidence of testicular teratomas [Krentz et al., 2009]. These tumors differ in a number of respects from the testicular germ cell tumors most common in humans [Oosterhuis and Looijenga, 2005]. However, genome-wide association studies have shown strong association of DMRT1 with human testicular germ cell cancer [Turnbull et al., 2010; Kanetsky et al., 2011; Kratz et al., 2011]. Gain of distal 9p, involving DMRT1, also is found in all cases of the rare germ cell cancer spermatocytic seminoma, which is found exclusively in older men [Looijenga et al., 2006]. These tumors are thought to arise from a different stage of germ cell development and may result from ectopic DMRT1 activity [Oosterhuis and Looijenga, 2005].

DMRT1 and Testis Development

\section{Insights from Mouse Conditional Genetics: DMRT1 and Somatic Sexual Cell Fate}

The role of DMRT1 in Sertoli cells is most germane to DSD. Dmrt1 null mutants or conditional mutants with Dmrt1 deleted in fetal bipotential progenitor cells have apparently normal testes at birth, but mutant Sertoli cells fail to complete differentiation and are unable to support spermatogenesis beyond meiotic prophase [Raymond et al., 2000; Kim et al., 2007a]. Unexpectedly, however, deletion of Dmrt1 in pre-Sertoli or Sertoli cells not only prevents the completion of differentiation but also causes their transdifferentiation to granulosa-like cells, the female supporting cell counterpart [Matson et al., 2011]. This sexual transdifferentiation occurs even when Dmrt1 is deleted in fully differentiated post-mitotic adult Sertoli cells, demonstrating that male sexual cell fate must be continuously maintained from at least puberty onward. Loss of Dmrt1 in Sertoli cells causes ectopic expression of key female sex regulators including Foxl2, Wnt4, Rspo1, Esr1, and Esr2, and genetic analysis indicates that these genes drive the male-to-female transdifferentiation that ensues [Minkina et al., 2014]. Whether the transdifferentiated granulosa-like cells are able to support oogenesis is unknown, because germ cells in the mutant XY gonad undergo apoptosis and efforts to co-transplant mutant $\mathrm{XY}$ somatic cells together with wild type XX oocytes have not been successful (D.Z., unpubl. data).

Why do Sertoli cells need a sex maintenance system? When Dmrt1 is deleted, the feminizing genes are activated by inappropriate retinoic acid (RA) signaling. Blocking RA signaling or deleting the RA receptor Rara suppresses this activation and largely blocks transdifferentiation [Minkina et al., 2014]. A likely model is that DMRT1 not only limits RA signaling in spermatogonia but also restricts the activity of RA in somatic cells, thereby allowing RA to promote meiosis in spermatocytes without causing premature meiotic initiation in spermatogonia or sexual cell fate transdifferentiation in Sertoli cells.

Granulosa cells also need sex maintenance: deletion of Foxl2 in the ovary causes a reciprocal, female-to-male, transdifferentiation. These results suggest that DMRT1 and FOXL2 anchor antagonistic regulatory networks that ensure the stability of somatic sex throughout postnatal life [Uhlenhaut et al., 2009]. Consistent with this view, deletion of Foxl2 suppresses transdifferentiation of XY Dmrt1 mutants [Minkina et al., 2014] and the transcriptome of XX gonads expressing ectopic Dmrt1 is very similar to that of XX gonads lacking Foxl2 [Lindeman et al., 2015]. Whether DMRT1 is required in the human gonad 
for the testis determination decision or subsequently for testis differentiation, and whether it maintains male sexual fate in the human gonad are unknown. This uncertainty is in part because early failure of human testicular development, for example in SRY or SOX9 mutants, results in highly dysgenic "streak" gonads and female internal and external genitalia, a condition known as Swyer Syndrome (MIM 400044).

DMRT1 not only is required to maintain male fate in the XY gonad but also can impose male fate on somatic cells of the XX gonad. Ectopic expression of DMRT1 in the ovary from a single copy transgene, starting either around E11.5 in the fetal gonad, driven by Sf1-Cre, or in the adult gonad, driven by UBC-CreERT2 or Hsd17b1$\mathrm{Cre}$, can reprogram granulosa cells into Sertoli-like cells organized into tubule-like structures [Lindeman et al., 2015] and can sexually transform the ovarian transcriptome (Lindeman and Zarkower, unpubl. data). These results indicate that DMRT1 can act instructively to determine male cell fate. However, transdifferentiation did not start until about 1-2 weeks after birth, similar to the timing of transdifferentiation in XY Dmrt1 mutants. Even expressing DMRT1 from about E9.5 using wt1-Cre did not cause fetal masculinization. This is perhaps unsurprising, given that DMRT1 normally is expressed at similar levels in bipotential Sertoli/granulosa progenitor cells of the 2 sexes prior to sex determination. One study has found that a multicopy Dmrt 1 cDNA driven by the wt 1 promoter can cause partial female-to-male sex reversal [Zhao et al., 2015]. However this observation involved progeny of a single transgenic founder with extremely high expression of Dmrt1 and some of its targets, in contrast to the approximately physiological DMRT1 expression level in the previous study. Also, a different multicopy transgenic study also expressing Dmrt1 from the wt1 promoter found no effect on sex determination [Agbor et al., 2013]. Possible explanations for the discrepancy are that highly overexpressed DMRT1 and/or its targets competes for regulatory cofactors shared with transcription factors involved in female sex determination, or that the wt1-Dmrt1 transgene in the single affected founder line was inserted in or near a locus involved in female gonadogenesis.

\section{The DM Domain and Gene Regulation}

DMRT proteins are site-specific DNA binding proteins, and genetic experiments have confirmed that they regulate transcription of target genes by activation or re- pression. The DMRT1 DNA-binding consensus sequence in vitro and in vivo is a 13-bp pseudopalindromic DNA motif, which is also bound by other DMRT proteins [Murphy et al., 2007]. DMRT proteins can form heteromers on DNA both in vitro and in vivo, raising the possibility that they may interact functionally by forming mixed complexes with different activities [Murphy et al., 2007; Zhang et al., 2014; Zhang and Zarkower, 2017]. Xray crystallography has revealed that the DM domain inserts its recognition helix into the DNA major groove and makes sequence-specific hydrogen bond contacts with key DNA bases in the manner of many other site-specific DNA binding proteins (Fig. 1c, d) [Murphy et al., 2015]. The DM domain can bind DNA as a trimer, with an antiparallel dimer of protomers on one side of the binding site and a single protomer on the other side. Unexpectedly, however, the antiparallel dimerized protomers jointly insert their recognition helices into the same segment of an inherently wide major groove. This binding mode, involving 2 helices contacting the same major groove segment, may be unique to the DM domain, as it has not been observed among the hundreds of other protein/DNA complexes structurally analyzed to date. Both in vitro and in vivo DMRT1 is able to bind DNA not only as a trimer but also as a dimer or tetramer, based on EMSA (see Fig. 2) and high resolution ChIP-exo analysis [Murphy et al., 2015]. The stoichiometry of binding depends both on the specific sequence and the inherent shape of the particular binding site.

Understanding how DMRT1 functions requires an understanding of how it can impose a new fate on cells even after differentiation is complete and how it can perform very different functions in different contexts, for example in somatic versus germ cells or in SSCs versus differentiating spermatogonia. There are several properties that could help account for these abilities. These properties include the differences in stoichiometry of DNA binding, interactions with cell type-specific transcriptional regulators, the ability to bind closed chromatin, effects on genome organization, or a combination of these. The significance of the alternative DNA binding stoichiometries remains unclear, as individual stoichiometries do not correspond in an obvious way with targets that are, for example, activated or repressed by DMRT1. R111 plays a key role in DNA base recognition (Fig. 1d), and the dominant change in DNA binding stoichiometry caused by the R111G mutation hints that context-dependent binding modes may be important. The other DSDassociated DMRT1 mutations have not yet been tested for such dominance. 
Fig. 3. Model of DMRT1 regulation of sexual cell fate maintenance and reprogramming. Top: In sexual cell fate maintenance, DMRT1 (purple) occupies distal regulatory elements, in many cases with SOX9 (blue) and other regulators (brown). This binding helps keep chromatin accessible ("open") and promotes expression of genes involved in Sertoli cell fate and function. Bottom: In reprogramming, DMRT1 binds inaccessible ("closed") chromatin and promotes its conversion to a form accessible to SOX9 and other regulators. Occupancy by DMRT1 and other regulators relieves repression and promotes activation of genes in involved in Sertoli cell fate and function. How DMRT1 affects chromatin accessibility and potentially chromatin interactions is unknown, as are whether DMRT1 binding stoichiometry affects these functions and whether DMRT1 binding can promote formation of repressive chromatin at granulosa-expressed genes.
Sexual cell fate maintenance:

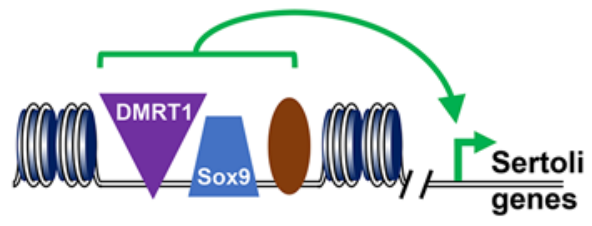

Sexual cell fate reprogramming:

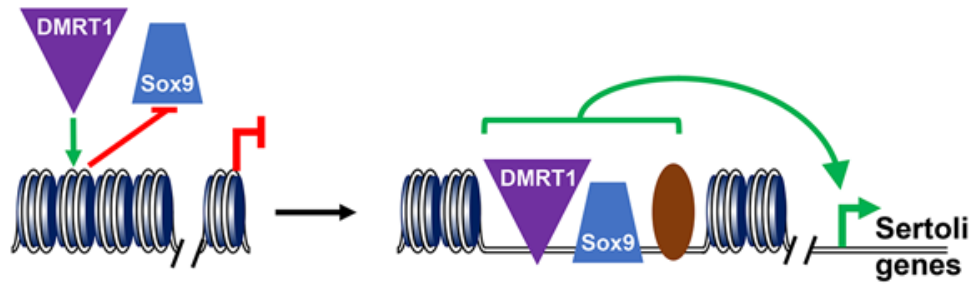

There is stronger evidence that some of the other properties are significant. Genetic and molecular analysis suggest that DMRT1 functionally interacts with SOX9 both in the fetal and adult testis. Deletion of Sox 9 causes more severe sexual transdifferentiation of Dmrt1 mutant testes [Minkina et al., 2014], and deletion of Sox9 with its close paralog Sox8 also limits reprogramming of granulosa cells by DMRT1 in the ovary [Lindeman et al., 2015, 2021]. ChIP-seq experiments show that DMRT1 and SOX9 often bind near one another on target genes in the fetal testis [Rahmoun et al., 2017], in juvenile Sertoli cells, and when expressed ectopically in the ovary [Lindeman et al., 2021], suggesting that they have joint effects on target gene expression. DMRT1 also functionally interacts with DMRT6 in spermatogonia, but here the relationship may be antagonistic [Zhang et al., 2014]. DMRT1 promotes spermatogonial differentiation and activates spermatogonial regulators such as Sohlh1, whereas DMRT6 is expressed late in spermatogonial development, in intermediate and B spermatogonia, and helps repress the mitotic and differentiation programs prior to meiosis by turning off spermatogonial regulators like Sohlh1 and Sohlh2, as well as Dmrt1. DMRT1 and DMRT6 bind to an overlapping set of genomic sites, and ChIP-reChIP shows that they can bind the same site at the same time on several key target genes, suggesting that they form heteromers in which the presence of DMRT6 may switch regulation from activation to repression.
DMRT1 appears to act as a pioneer transcription factor, binding "closed" low-modification chromatin and opening it to binding by other transcriptional regulators [Lindeman et al., 2021]. In granulosa cells, ectopic DMRT1 can bind differentially accessible regions (DARs) that normally are open in Sertoli cells but closed in granulosa cells. Many of these sites are not efficiently bound by SOX9 when it is expressed alone, but expression of DMRT1 with SOX9 allows binding by SOX9, consistent with DMRT1 pioneering for SOX9 and presumably other regulatory factors [Lindeman et al., 2021]. Pioneer function requires the ability to bind nucleosomal DNA and, while this has not yet been demonstrated for DMRT1 in vitro, structural modeling suggests that DMRT1 should be able to interact with its site in a nucleosomal context. Finally, DNA binding by DMRT1 as a multimer suggests the possibility that it could mediate chromatin contacts that could help form functional domains. Preliminary $\mathrm{HiC}$ data indicate that ectopic DMRT1 does result in formation of new chromatin loops (M.W.M and D.Z., unpubl. data), but further work will be required to determine their significance for gene expression and how directly DMRT1 participates in the formation of these structures. A model for how DMRT1 controls maintenance and reprogramming of sexual cell fate is shown in Figure 3. 


\section{Epilogue: Open Questions and Future Directions}

The past 2 decades have revealed much about $D M R T$ genes in general and $D M R T 1$ in particular. We now have convincing evidence that $D M R T 1$ is an important regulator of testicular differentiation in most or all vertebrates, is involved in the evolution of new vertebrate sex determination mechanisms, has a number of distinct functions in the mouse gonad both during and after development, and is a key locus involved in 9p deletion-associated DSD. We also are gaining a more detailed view of how DMRT1 engages with target genes, know the identities of many of these genes, and are beginning to identify other regulators that cooperate with DMRT1 to control gonadal gene expression.

Much remains to be learned, however. Among the open questions relating to the role of DMRT1 in DSD are whether DMRT1 controls sex determination or sex differentiation in the human gonad (or both), how genetic background intersects with DMRT1 loss in DSD, whether male sex maintenance exists in the human testis, and whether other genes on distal $9 \mathrm{p}$ contribute to testicular differentiation or male fertility. Most of these are questions not easily resolved using mice or other model organisms, but advances in organoid technology may soon accelerate progress [Sakib et al., 2019].

\section{Acknowledgements}

D.Z. thanks Vivian Bardwell for 25 years of partnership studying $D M R T$ genes and thanks members of their research group over that span, as well as many colleagues worldwide, for their collegiality, insights, and generosity with ideas and reagents in the shared endeavor to better understand mammalian gonadogenesis and sexual dimorphism. The authors also thank Vivian Bardwell for assistance with figures.

\section{Conflict of Interest Statement}

The authors have no conflicts of interest to declare.

\section{Funding Sources}

Work in the Zarkower and Bardwell laboratory has been supported by grants from the NIH (GM059152 and GM127379) and by the University of Minnesota Foundation and University of Minnesota Medical School.

\section{Author Contributions}

D.Z. wrote the manuscript and M.W.M. performed EMSA experiments and edited the manuscript.

\section{References}

Agbor VA, Tao S, Lei N, Heckert LL. A Wt1Dmrt1 transgene restores DMRT1 to Sertoli cells of Dmrt1(-/-) testes: a novel model of DMRT1-deficient germ cells. Biol Reprod. 2013;88:51.

Alfi OS, Donnell GN, Allderdice PW, Derencsenyi A. The 9p- syndrome. Ann Genet. 1976; 19:11-6.

Andersson LS, Larhammar M, Memic F, Wootz $\mathrm{H}$, Schwochow D, Rubin CJ, et al. Mutations in DMRT3 affect locomotion in horses and spinal circuit function in mice. Nature. 2012; 488:642-6.

Baker BS, Ridge KA. Sex and the single cell. I. On the action of major loci affecting sex determination in Drosophila melanogaster. Genetics. 1980;94:383-423.

Balciuniene J, Bardwell VJ, Zarkower D. Mice mutant in the DM domain gene Dmrt4 are viable and fertile but have polyovular follicles. Mol Cell Biol. 2006;26:8984-91.

Barrionuevo F, Bagheri-Fam S, Klattig J, Kist R, Taketo MM, Englert C, et al. Homozygous inactivation of Sox9 causes complete XY sex reversal in mice. Biol Reprod. 2006;74:195-201.
Bartels I, Pütz I, Reintjes N, Netzer C, Shoukier M. Normal intelligence and premature ovarian failure in an adult female with a 7.6 Mb de novo terminal deletion of chromosome $9 \mathrm{p}$. Eur J Med Genet. 2013;56:458-62.

Bennett CP, Docherty Z, Robb SA, Ramani P, Hawkins JR, Grant D. Deletion 9p and sex reversal. J Med Genet. 1993;30:518-20.

Bewick AJ, Anderson DW, Evans BJ. Evolution of the closely related, sex-related genes DM-W and DMRT1 in African clawed frogs (Xenopus). Evolution. 2011;65:698-712.

Buonocore F, Clifford-Mobley O, King TFJ, Striglioni N, Man E, Suntharalingham JP, et al. Next-generation sequencing reveals novel genetic variants (SRY, DMRT1, NR5A1, DHH, DHX37) in adults with 46,XY DSD. J Endocr Soc. 2019;3:2341-60.

Burtis KC, Baker BS. Drosophila doublesex gene controls somatic sexual differentiation by producing alternatively spliced mRNAs encoding related sex-specific polypeptides. Cell. 1989;56:997-1010.

Calvari V, Bertini V, De Grandi A, Peverali G, Zuffardi O, Ferguson-Smith M, et al. A new submicroscopic deletion that refines the $9 \mathrm{p}$ region for sex reversal. Genomics. 2000;65:203-12.
Chauhan V, Jyotsna VP, Jain V, Khadgawat R, Dada R. Novel heterozygous genetic variants in patients with $46, \mathrm{XY}$ gonadal dysgenesis. Horm Metab Res. 2017;49:36-42.

Chong T, Collins JJ 3rd, Brubacher JL, Zarkower D, Newmark PA. A sex-specific transcription factor controls male identity in a simultaneous hermaphrodite. Nat Commun. 2013;4: 1814.

Crocker M, Coghill SB, Cortinho R. An unbalanced autosomal translocation $(7 ; 9)$ associated with feminization. Clin Genet. 1988;34: 70-3.

De Clercq S, Keruzore M, Desmaris E, Pollart C, Assimacopoulos S, Preillon J, et al. DMRT5 together with DMRT3 directly controls hippocampus development and neocortical area map formation. Cereb Cortex. 2018;28:493509.

Desmaris E, Keruzore M, Saulnier A, Ratié L, Assimacopoulos S, De Clercq S, et al. DMRT5, DMRT3, and EMX2 cooperatively repress Gsx2 at the pallium-subpallium boundary to maintain cortical identity in dorsal telencephalic progenitors. J Neurosci. 2018;38:9105-21. 
Eggers S, Sadedin S, van den Bergen JA, Robevska G, Ohnesorg T, Hewitt J, et al. Disorders of sex development: insights from targeted gene sequencing of a large international patient cohort. Genome Biol. 2016;17:243.

Erdman SE, Burtis KC. The Drosophila doublesex proteins share a novel zinc finger related DNA binding domain. EMBO J. 1993;12: 527-35.

Erdman SE, Chen HJ, Burtis KC. Functional and genetic characterization of the oligomerization and DNA binding properties of the Drosophila doublesex proteins. Genetics. 1996; 144:1639-52.

Fahrioglu U, Murphy MW, Zarkower D, Bardwell VJ. mRNA expression analysis and the molecular basis of neonatal testis defects in Dmrt1 mutant mice. Sex Dev. 2007;1:42-58.

Fan Y, Zhang X, Wang L, Wang R, Huang Z, Sun $Y$, et al. Diagnostic application of targeted next-generation sequencing of 80 genes associated with disorders of sexual development. Sci Rep. 2017;7:44536.

Flejter WL, Fergestad J, Gorski J, Varvill T, Chandrasekharappa S. A gene involved in XY sex reversal is located on chromosome 9 , distal to marker D9S1779. Am J Hum Genet. 1998;63: 794-802.

Foster JW, Dominguez-Steglich MA, Guioli S, Kwok C, Weller PA, Stevanović M, et al. Campomelic dysplasia and autosomal sex reversal caused by mutations in an SRY-related gene. Nature. 1994;372:525-30.

Fryns JP, Kleczkowska A, Casaer P, van den Berghe H. Double autosomal chromosomal aberration ( $3 p$ trisomy $/ 9 p$ monosomy) and sexreversal. Ann Genet. 1986;29:49-52.

Fujimoto Y, Okuyama T, Iijima M, Tanaka T, Horikawa R, Yamada K, et al. Genitourinary phenotype in XX patients with distal 9p monosomy. Mol Genet Metab. 2004;82:173-9.

Ge C, Ye J, Zhang H, Zhang Y, Sun W, Sang Y, et al. Dmrt1 induces the male pathway in a turtle species with temperature-dependent sex determination. Development. 2017;144:222233.

Geuverink E, Beukeboom LW. Phylogenetic distribution and evolutionary dynamics of the sex determination genes doublesex and transformer in insects. Sex Dev. 2014;8:38-49.

Guioli S, Schmitt K, Critcher R, Bouzyk M, Spurr NK, Ogata T, et al. Molecular analysis of $9 \mathrm{p}$ deletions associated with XY sex reversal: refining the localization of a sex-determining gene to the tip of the chromosome. Am J Hum Genet. 1998;63:905-8.

Guo J, Grow EJ, Mlcochova H, Maher GJ, Lindskog $\mathrm{C}, \mathrm{Nie} \mathrm{X}$, et al. The adult human testis transcriptional cell atlas. Cell Res. 2018;28: 1141-57.

Hoo JJ, Salefsky IS, Lin CC. Possible localisation of a recessive testis forming gene on 9p24. Am J Hum Genet. 1988;45(Suppl 1):A78.

Huret JL, Leonard C, Forestier B, Rethoré MO, Lejeune J. Eleven new cases of del(9p) and features from 80 cases. J Med Genet. 1988;25: 741-9.
Inui M, Tamano M, Kato T, Takada S. CRISPR/ Cas9-mediated simultaneous knockout of Dmrt1 and Dmrt3 does not recapitulate the $46, \mathrm{XY}$ gonadal dysgenesis observed in $9 \mathrm{p} 24.3$ deletion patients. Biochem Biophys Rep. 2017;9:238-44.

Jorgensen A, Nielsen JE, Blomberg Jensen M, Graem N, Rajpert-De Meyts E. Analysis of meiosis regulators in human gonads: a sexually dimorphic spatio-temporal expression pattern suggests involvement of DMRT1 in meiotic entry. Mol Hum Reprod. 2012;18: 523-34.

Jotterand M, Juillard E. A new case of trisomy for the distal part of $13 \mathrm{q}$ due to maternal translocation, $\mathrm{t}(9 ; 13)(\mathrm{p} 21 ; \mathrm{q} 21)$. Hum Genet. 1976; 33:213-22.

Kanetsky PA, Mitra N, Vardhanabhuti S, Vaughn DJ, Li M, Ciosek SL, et al. A second independent locus within DMRT1 is associated with testicular germ cell tumor susceptibility. Hum Mol Genet. 2011;20:3109-17.

Kato Y, Kobayashi K, Watanabe H, Iguchi T. Environmental sex determination in the branchiopod crustacean Daphnia magna: deep conservation of a Doublesex gene in the sexdetermining pathway. PLoS Genet. 2011;7: e1001345.

Kawamata M, Nishimori K. Mice deficient in Dmrt7 show infertility with spermatogenic arrest at pachytene stage. FEBS Lett. 2006;580: 6442-6.

Kim S, Kettlewell JR, Anderson RC, Bardwell VJ, Zarkower D. Sexually dimorphic expression of multiple doublesex-related genes in the embryonic mouse gonad. Gene Expr Patterns. 2003;3:77-82.

Kim S, Bardwell VJ, Zarkower D. Cell type-autonomous and non-autonomous requirements for Dmrt1 in postnatal testis differentiation. Dev Biol. 2007a;307:314-27.

Kim S, Namekawa SH, Niswander LM, Ward JO, Lee JT, Bardwell VJ, et al. A mammal-specific Doublesex homolog associates with male sex chromatin and is required for male meiosis. PLoS Genet. 2007b;3:e62.

Kondo M, Nanda I, Hornung U, Schmid M, Schartl M. Evolutionary origin of the medaka Y chromosome. Curr Biol. 2004;14:1664-9.

Konno D, Iwashita M, Satoh Y, Momiyama A, Abe T, Kiyonari $\mathrm{H}$, et al. The mammalian DM domain transcription factor Dmrta2 is required for early embryonic development of the cerebral cortex. PLoS One. 2012;7:e46577.

Kratz CP, Han SS, Rosenberg PS, Berndt SI, Burdett $\mathrm{L}$, Yeager $\mathrm{M}$, et al. Variants in or near KITLG, BAK1, DMRT1, and TERT-CLPTM1L predispose to familial testicular germ cell tumour. J Med Genet. 2011;48:473-6.

Krentz AD, Murphy MW, Kim S, Cook MS, Capel $\mathrm{B}$, Zhu R, et al. The DM domain protein DMRT1 is a dose-sensitive regulator of fetal germ cell proliferation and pluripotency. Proc Natl Acad Sci USA. 2009;106:22323-8.
Krentz AD, Murphy MW, Sarver AL, Griswold MD, Bardwell VJ, Zarkower D. DMRT1 promotes oogenesis by transcriptional activation of Stra8 in the mammalian fetal ovary. Dev Biol. 2011;356:63-70.

Lambeth LS, Raymond CS, Roeszler KN, Kuroiwa A, Nakata T, Zarkower D, et al. Over-expression of DMRT1 induces the male pathway in embryonic chicken gonads. Dev Biol. 2014; 389:160-72.

Ledig S, Hiort O, Scherer G, Hoffmann M, Wolff $\mathrm{G}$, Morlot S, et al. Array-CGH analysis in patients with syndromic and non-syndromic $\mathrm{XY}$ gonadal dysgenesis: evaluation of array CGH as diagnostic tool and search for new candidate loci. Hum Reprod. 2010;25:263746.

Ledig S, Hiort O, Wünsch L, Wieacker P. Partial deletion of DMRT1 causes 46,XY ovotesticular disorder of sexual development. Eur J Endocrinol. 2012;167:119-24.

Lei N, Hornbaker KI, Rice DA, Karpova T, Agbor VA, Heckert LL. Sex-specific differences in mouse DMRT1 expression are both cell typeand stage-dependent during gonad development. Biol Reprod. 2007;77:466-75.

Lindeman RE, Gearhart MD, Minkina A, Krentz AD, Bardwell VJ, Zarkower D. Sexual cell-fate reprogramming in the ovary by DMRT1. Curr Biol. 2015;25:764-71.

Lindeman RE, Murphy MW, Agrimson KS, Gewiss RL, Bardwell VJ, Gearhart MD, et al. The conserved sex regulator DMRT1 recruits SOX9 in sexual cell fate reprogramming. $\mathrm{Nu}-$ cleic Acids Res2021;49:6144-64.

Looijenga LH, Hersmus R, Gillis AJ, Pfundt R, Stoop HJ, van Gurp RJ, et al. Genomic and expression profiling of human spermatocytic seminomas: primary spermatocyte as tumorigenic precursor and DMRT1 as candidate chromosome 9 gene. Cancer Res. 2006;66: 290-302.

Lopes AM, Aston KI, Thompson E, Carvalho F, Gonçalves J, Huang N, et al. Human spermatogenic failure purges deleterious mutation load from the autosomes and both sex chromosomes, including the gene DMRT1. PLoS Genet. 2013;9:e1003349.

Mason DA, Rabinowitz JS, Portman DS. Dmd-3, a doublesex-related gene regulated by tra- 1 , governs sex-specific morphogenesis in C. elegans. Development. 2008;135:2373-82.

Matson CK, Zarkower D. Sex and the singular DM domain: insights into sexual regulation, evolution and plasticity. Nat Rev Genet. 2012; 13:163-74.

Matson CK, Murphy MW, Griswold MD, Yoshida S, Bardwell VJ, Zarkower D. The mammalian doublesex homolog DMRT1 is a transcriptional gatekeeper that controls the mitosis versus meiosis decision in male germ cells. Dev Cell. 2010;19:612-24.

Matson CK, Murphy MW, Sarver AL, Griswold MD, Bardwell VJ, Zarkower D. DMRT1 prevents female reprogramming in the postnatal mammalian testis. Nature. 2011;476:101-4. 
Matsuda M, Nagahama Y, Shinomiya A, Sato T, Matsuda C, Kobayashi T, et al. DMY is a Yspecific DM-domain gene required for male development in the medaka fish. Nature. 2002;417:559-63.

Matsuda M, Sato T, Toyazaki Y, Nagahama Y, Hamaguchi S, Sakaizumi M. Oryzias curvinotus has DMY, a gene that is required for male development in the medaka, O. latipes. Zoo$\log$ Sci. 2003;20:159-61.

McDonald MT, Flejter W, Sheldon S, Putzi MJ, Gorski JL. XY sex reversal and gonadal dysgenesis due to 9p24 monosomy. Am J Med Genet. 1997;73:321-6.

Minkina A, Matson CK, Lindeman RE, Ghyselinck NB, Bardwell VJ, Zarkower D. DMRT1 protects male gonadal cells from retinoid-dependent sexual transdifferentiation. Dev Cell. 2014;29:511-20.

Muroya K, Okuyama T, Goishi K, Ogiso Y, Fukuda S, Kameyama J, et al. Sex-determining gene(s) on distal 9p: clinical and molecular studies in six cases. J Clin Endocrinol Metab. 2000;85:3094-100.

Murphy MW, Zarkower D, Bardwell VJ. Vertebrate DM domain proteins bind similar DNA sequences and can heterodimerize on DNA. BMC Mol Biol. 2007;8:58.

Murphy MW, Lee JK, Rojo S, Gearhart MD, Kurahashi K, Banerjee S, et al. An ancient protein-DNA interaction underlying metazoan sex determination. Nat Struct Mol Biol. 2015; 22:442-51.

Nakagawa T, Zhang T, Kushi R, Nakano S, Endo $\mathrm{T}$, Nakagawa M, et al. Regulation of mitosismeiosis transition by the ubiquitin ligase $\beta$-TrCP in male germ cells. Development. 2017;144:4137-47.

Nanda I, Shan Z, Schartl M, Burt DW, Koehler M, Nothwang H, et al. 300 million years of conserved synteny between chicken $\mathrm{Z}$ and human chromosome 9. Nat Genet. 1999;21:258-9.

Nanda I, Kondo M, Hornung U, Asakawa S, Winkler C, Shimizu A, et al. A duplicated copy of DMRT1 in the sex-determining region of the Y chromosome of the medaka, Oryzias latipes. Proc Natl Acad Sci USA. 2002;99: 11778-83.

Ogata T, Muroya K, Matsuo N, Hata J, Fukushima Y, Suzuki Y. Impaired male sex development in an infant with molecularly defined partial 9p monosomy: implication for a testis forming gene(s) on 9p. J Med Genet. 1997;34:3314.

Ogata T, Muroya $\mathrm{K}$, Ohashi $\mathrm{H}$, Mochizuki $\mathrm{H}$, Hasegawa T, Kaji M. Female gonadal development in XX patients with distal 9p monosomy. Eur J Endocrinol. 2001;145:613-7.

Oosterhuis JW, Looijenga LH. Testicular germcell tumours in a broader perspective. Nat Rev Cancer. 2005;5:210-22.

Ottolenghi C, McElreavey K. Deletions of $9 p$ and the quest for a conserved mechanism of sex determination. Mol Genet Metab. 2000;71: 397-404.
Ottolenghi C, Veitia R, Quintana-Murci L, Torchard D, Scapoli L, Souleyreau-Therville N, et al. The region on $9 \mathrm{p}$ associated with $46, \mathrm{XY}$ sex reversal contains several transcripts expressed in the urogenital system and a novel doublesex-related domain. Genomics. 2000;64:1708

Ottolenghi C, Fellous M, Barbieri M, McElreavey K. Novel paralogy relations among human chromosomes support a link between the phylogeny of doublesex-related genes and the evolution of sex determination. Genomics. 2002;79:333-43.

Ounap K, Uibo O, Zordania R, Kiho L, Ilus T, Oiglane-Shlik E, et al. Three patients with $9 \mathrm{p}$ deletions including DMRT1 and DMRT2: a girl with XY complement, bilateral ovotestes, and extreme growth retardation, and two XX females with normal pubertal development. Am J Med Genet A. 2004;130A:415-23.

Parker KL, Schimmer BP, Schedl A. Genes essential for early events in gonadal development. Cell Mol Life Sci. 1999;55:831-8.

Quinonez SC, Park JM, Rabah R, Owens KM, Yashar BM, Glover TW, et al. 9p partial monosomy and disorders of sex development: review and postulation of a pathogenetic mechanism. Am J Med Genet A. 2013; 161A:1882-96.

Rahmoun M, Lavery R, Laurent-Chaballier S, Bellora N, Philip GK, Rossitto M, et al. In mammalian foetal testes, SOX9 regulates expression of its target genes by binding to genomic regions with conserved signatures. Nucleic Acids Res. 2017;45:7191-211.

Raymond CS, Shamu CE, Shen MM, Seifert KJ, Hirsch B, Hodgkin J, et al. Evidence for evolutionary conservation of sex-determining genes. Nature. 1998;391:691-5.

Raymond CS, Kettlewell JR, Hirsch B, Bardwell VJ, Zarkower D. Expression of Dmrt1 in the genital ridge of mouse and chicken embryos suggests a role in vertebrate sexual development. Dev Biol. 1999a;215:208-20.

Raymond CS, Parker ED, Kettlewell JR, Brown LG, Page DC, Kusz K, et al. A region of human chromosome $9 \mathrm{p}$ required for testis development contains two genes related to known sexual regulators. Hum Mol Genet. 1999b;8: 989-96.

Raymond CS, Murphy MW, O'Sullivan MG, Bardwell VJ, Zarkower D. Dmrtl, a gene related to worm and fly sexual regulators, is required for mammalian testis differentiation. Genes Dev. 2000;14:2587-95.

Robinett CC, Vaughan AG, Knapp JM, Baker BS. Sex and the single cell. II. There is a time and place for sex. PLoS Biol. 2010;8:e1000365.

Sakib S, Voigt A, Goldsmith T, Dobrinski I. Three-dimensional testicular organoids as novel in vitro models of testicular biology and toxicology. Environ Epigenet. 2019;5:dvz011.

Saulnier A, Keruzore M, De Clercq S, Bar I, Moers $\mathrm{V}$, Magnani D, et al. The doublesex homolog Dmrt5 is required for the development of the caudomedial cerebral cortex in mammals. Cereb Cortex2013;23:2552-67.
Seo KW, Wang Y, Kokubo H, Kettlewell JR, Zarkower DA, Johnson RL. Targeted disruption of the DM domain containing transcription factor Dmrt2 reveals an essential role in somite patterning. Dev Biol. 2006;290:200-10.

Shen MM. Hodgkin J: mab-3, a gene required for sex-specific yolk protein expression and a male-specific lineage in C. elegans. Cell. 1988; 54:1019-31.

Smith CA, Roeszler KN, Ohnesorg T, Cummins DM, Farlie PG, Doran TJ, et al. The avian Zlinked gene DMRT1 is required for male sex determination in the chicken. Nature. 2009; 461:267-71.

Tewes AC, Ledig S, Tüttelmann F, Kliesch S, Wieacker P. DMRT1 mutations are rarely associated with male infertility. Fertil Steril. 2014;102:816-e3.

Turnbull C, Rapley EA, Seal S, Pernet D, Renwick A, Hughes D, et al. Variants near DMRT1, TERT and ATF7IP are associated with testicular germ cell cancer. Nat Genet. 2010;42: 604-7.

Uhlenhaut NH, Jakob S, Anlag K, Eisenberger T, Sekido R, Kress J, et al. Somatic sex reprogramming of adult ovaries to testes by FOXL2 ablation. Cell. 2009;139:1130-42.

Vallstedt A, Kullander K. Dorsally derived spinal interneurons in locomotor circuits. Ann N Y Acad Sci. 2013;1279:32-42.

Veith AM, Klattig J, Dettai A, Schmidt C, Englert C, Volff JN. Male-biased expression of Xchromosomal DM domain-less Dmrt8 genes in the mouse. Genomics. 2006;88:185-95.

Veitia R, Nunes M, Brauner R, Doco-Fenzy M, Joanny-Flinois $\mathrm{O}$, Jaubert $\mathrm{F}$, et al. Deletions of distal $9 \mathrm{p}$ associated with $46, \mathrm{XY}$ male to female sex reversal: definition of the breakpoints at 9p23.3-p24.1. Genomics. 1997;41:271-4.

Veitia RA, Nunes M, Quintana-Murci L, Rappaport R, Thibaud E, Jaubert F, et al. Swyer syndrome and 46,XY partial gonadal dysgenesis associated with $9 p$ deletions in the absence of monosomy-9p syndrome. Am J Hum Genet. 1998;63:901-5.

Vialard F, Ottolenghi C, Gonzales M, Choiset A, Girard S, Siffroi JP, et al. Deletion of 9p associated with gonadal dysfunction in $46, \mathrm{XY}$ but not in 46,XX human fetuses. J Med Genet. 2002;39:514-8

Volff JN, Zarkower D, Bardwell VJ, Schartl M. Evolutionary dynamics of the DM domain gene family in metazoans. J Mol Evol. 2003; 57(Suppl 1):S241-9.

Wagner T, Wirth J, Meyer J, Zabel B, Held M, Zimmer J, et al. Autosomal sex reversal and campomelic dysplasia are caused by mutations in and around the SRY-related gene SOX9. Cell. 1994;79:1111-20.

Wang M, Liu X, Chang G, Chen Y, An G, Yan L, et al. Single-cell RNA sequencing analysis reveals sequential cell fate transition during human spermatogenesis. Cell Stem Cell. 2018; 23:599-e4. 
Webster KA, Schach U, Ordaz A, Steinfeld JS, Draper BW, Siegfried KR. Dmrt1 is necessary for male sexual development in zebrafish. Dev Biol. 2017;422:33-46.

Wexler JR, Plachetzki DC, Kopp A. Pan-metazoan phylogeny of the DMRT gene family: a framework for functional studies. Dev Genes Evol. 2014;224:175-81.

Yi W, Zarkower D. Similarity of DNA binding and transcriptional regulation by Caenorhabditis elegans MAB-3 and Drosophila melanogaster DSX suggests conservation of sex determining mechanisms. Development. 1999; $126: 873-81$.
Yoshimoto S, Okada E, Umemoto H, Tamura K, Uno Y, Nishida-Umehara C, et al. A W-linked DM-domain gene, DM-W, participates in primary ovary development in Xenopus laevis. Proc Natl Acad Sci USA. 2008;105:246974.

Zarkower D. DMRT genes in vertebrate gametogenesis. Curr Top Dev Biol. 2013;102:327-56.

Zhang T, Zarkower D. DMRT proteins and coordination of mammalian spermatogenesis. Stem Cell Res. 2017;24:195-202.

Zhang T, Murphy MW, Gearhart MD, Bardwell VJ, Zarkower D. The mammalian Doublesex homolog DMRT6 coordinates the transition between mitotic and meiotic developmental programs during spermatogenesis. Development. 2014;141:3662-71.
Zhang T, Oatley J, Bardwell VJ, Zarkower D. DMRT1 is required for mouse spermatogonial stem cell maintenance and replenishment. PLoS Genet. 2016;12:e1006293.

Zhao L, Svingen T, Ng ET, Koopman P. Femaleto-male sex reversal in mice caused by transgenic overexpression of Dmrt1. Development. 2015;142:1083-8.

Zhu L, Wilken J, Phillips NB, Narendra U, Chan G, Stratton SM, et al. Sexual dimorphism in diverse metazoans is regulated by a novel class of intertwined zinc fingers. Genes Dev. 2000; 14:1750-64. 